\title{
Robust Fuzzy Fault Detection for Non-Linear Stochastic Dynamic Systems
}

\author{
Magdy G. El-ghatwary ${ }^{1, *}$ and Steven X. Ding ${ }^{2}$ \\ ${ }^{1}$ Industrial Electronic and Automatic Control Department, Faculty of Electrical Engineering, Menoufia University,
Menouf 32952, Menofia, Egypt
${ }^{2}$ Institute for Automatic Control and Complex System, University of Duisburg-Essen, Germany
}

Abstract: One of the difficults for fault detection techniques for non-linear stochastic systems via model-based methods is the design of residual generation. In this paper, a new fault detection (FD) approche for non-linear stochastic systems is proposed. The non-linear system is represented by a discrite Takagi-Sugeno (TS) fuzzy model. The use of (TS) theory allows to represent non-linear systems as a set of linear systems, which represent the local system behavior around different operating points. The global system behaviour is described by a fuzzy fusion of all systems. The FD system for each local sub system is designed by solving the corresponding Discrite Algebra Recati Equation (DARE). Optimization algorithm based on minimizing the residual covariance matrix is used to obtain a robust FD for global system behavior. The observer gain matrices are solved using a set of Linear matrix Inequalities (LMIs).

\section{INTRODUCTION}

Over the past two decades, fault detection (FD) system has made a significant progress and recived considerable attension in both research and application domain. It leads to robust FD system for technical processes that can be modelled as linear time invarient (LTI) systems. If LTI system contains measurement noise, kalman filter is used to design FD system [1,2]. Recently Takagi-Sugeno (TS) fuzzy model was developed successfully to investigate non-linear system [3]. The robust fuzzy observer was discussed for TS fuzzy system with parameter uncertainties [4]. For systems with measurement disturbances, new descriptor observer approach was developed [5-7]. Extended kalman filter design method based on basic of adaptive fuzzy logic is shown in [8]. Robust fault estimation approach for vehicle lateral dynamic model is shown in [9]. In this paper, another fault detection approach is used. The proposed approach use fuzzy logic basics to design kalman filter for each fuzzy subsystem by solving the corresponding Discrite Algebra Recati Equation (DARE). Optimization algorithm based on minimizing the residual covariance matrix is used to obtain a robust FD for global system. The generated filter is robust against stochastic noise and sensitive with respect to faults.

This paper is organized as follows: Section 2 shows some prelimininaries about Takagi-sugeno (TS) fuzzy model, fault generation and fault evaluation, the proposed approache is presented in section 3; an application example is found in section 4; the conclusions are given in section 5 .

\section{PRELIMINARIES}

Some concepts relevant to this work are reviewed. First, the TS fuzzy model for non-linear system is present. Then

*Address correspondence to this author at the Industrial Electronic and Automatic Control Department, Faculty of Electrical Engineering, Menoufia University, Menouf 32952, Menofia, Egypt; Tel: \& Fax: +20483660716; E-mail: magdyelghatwary@yahoo.com residual generation and residual evaluation concepts are briefly described.

\subsection{TS Fuzzy Model Construction}

The fuzzy model proposed by Takagi and Sugeno is described by fuzzy IF-THEN rules, which represent local linear input-output relations of a non-linear system [3]. In order to consider stochastic noises and faults in the discrete TS fuzzy systems, we propose the discrete TS fuzzy system in which the ${ }^{i}$ th rule is formulated in the following form:

Rule $i$ : IF $z_{1}$ is $M_{i 1}$ and... and $z_{\theta}$ is $M_{i \theta}$ Then

$x(k+1)=A_{i} x(k)+B_{i} u(k)+E_{n, i} n(k)+E_{f, i} f(k)$

$y(k)=C i x(k)+D_{i} u(k)+F_{n, i} n(k)+F_{f, i} f(k)$

where $M_{i j}(i=1,2, \ldots, \mathrm{p}, j=1, \cdots, \theta)$ are fuzzy sets, $\mathrm{z}$ $=\left[z_{1}, \ldots, z_{\theta}\right]$ are premise variables, $x(k) \in \mathscr{R}^{n}$ is state vector, $u(k) \in \mathscr{R}^{p}$ and $y(k) \in \mathscr{R}^{q}$ are the input and measured output vectors respectively, $n(k) \in \mathscr{R}^{m}$ vector of zero mean white Gaussian noises with positive definite covariance matrix $\Sigma_{n}$, $f(k) \in \mathscr{R}_{l}^{l}$ is the fault to be detected. $A_{i}, B_{i}, E_{n, i}, E_{f, i}, C_{i}, D_{i}$, $F_{n, i}, F_{f, i}$ are known matrices with appropriate dimension. The defuzzified output of TS fuzzy system (1) is represented as:

$$
\begin{array}{r}
x(k+1)=\sum_{i=1}^{p} \mu_{i}\left[A_{i} x(k)+B_{i} u(k)+E_{n, i} n(k)+F_{f, i} f(k)\right] \\
y(k)=\sum_{i=1}^{p} \mu_{i}\left[C_{i} x(k)+D_{i} u(k)+F_{n, i} n(k)+F_{f, i} f(k)\right]
\end{array}
$$

where $\mu_{i}(z(k))=\frac{h_{i}(z(k))}{\sum_{i=1}^{p} h_{i}(z(k))}, \quad h_{i}(z(k))=\prod_{j=1}^{\theta} M_{i j}\left(z_{j}(k)\right)$.

$M_{i j}\left(z_{j}(k)\right) \geq 0$ is the grade of membership of $z_{j}(k)$ in $M_{i j}$. Assume that $\sum_{i=1}^{p} \prod_{j=1}^{\theta} M_{i j}\left(z_{j}(k)\right) \geq 0$. We have 


$$
\forall k \sum_{i=1}^{p} h_{i}(z(k))=1
$$

For simplifying notation $\mu_{i}(z(k))$ is replaced by $\mu_{i}$, which will also be adopted in the sequent sections if no confusion is caused.

Under the assumption that the current error is independent of the current noise, we provide

$$
\mathrm{E}\left[\mathrm{e}_{k} n_{i}^{T}\right]=E\left[n_{k} e_{i}^{T}\right]=0 \text {, for all } \mathrm{i}, \mathrm{k} .
$$

\subsection{Problem Formulation}

Fault detection problem can be formulated as design fault detection system which is robust with respect stochastic noises and sensitive with respect to faults.

\subsection{Residual Generation}

The first step to achieve robust FD system is to generate residual signal which is decoupled from the input signal $u(k)$. In this case, we consider the so-called TS fuzzy filter which is described as follows:

Rule $i$ : If $z_{1}$ is $M_{i 1}$ and... and $z_{\theta}$ is $M_{i \theta}$ Then

$$
\begin{gathered}
\hat{x}(k+1)=A_{i} \hat{x}(k)+B_{i} u(k)+\left(L_{i}^{*}+\Delta L_{i}\right)[y(k)-\hat{y}(k)] \\
\hat{y}(k)=C_{i} \hat{x}(k)+D_{i} u(k)
\end{gathered}
$$

where $L_{i}^{*}$ is the filter gain matrix for sub-model $i$ obtained from solving DARE for each local system, $\Delta L_{i}$ is increment in gain matrices obtained from reducing covariance matrix of residual signal. The fuzzy filter based residual generator is inferred as the weighted sum

$$
\begin{gathered}
\hat{x}(k+1)=\sum_{i=1}^{p} \mu_{i}\left[A_{i} \hat{x}(k)+B_{i} u(k)+\left(L_{i}^{*}+\Delta L_{i}\right)(y(k)-\hat{y}(k))\right] \\
\left.\hat{y}(k)=\sum_{i=1}^{p} \mu_{i}\left[C_{i} \hat{x}(k)+D_{i} u(k)\right)\right],
\end{gathered}
$$

where $\mu_{i}$ is the same weight function used in TS model (2). To analyze the convergence of the filter, the state error vector $e(k)=x(k)-\hat{x}(k)$ is given by the following difference equation.

$$
\begin{gathered}
e(k+1)=\sum_{i=1}^{p} \sum_{j=1}^{p} \mu_{i} \mu_{j}\left[\left(A_{i}-\left(L_{i}^{*}+\Delta L_{i}\right) C_{j}\right) e(k)\right. \\
\left.+\left(E_{n, i}-\left(L_{i}^{*}+\Delta L_{i}\right) F_{n, j}\right) n(k)+\left(E_{f, i}-\left(L_{i}^{*}+\Delta L_{i}\right) F_{f, j}\right) f(k)\right] \\
r(k)=\sum_{i=1}^{p} \mu_{1}\left[C_{i} e(k)+F_{n, i} n(k)+F_{f, i} f(k)\right],
\end{gathered}
$$

where $r(k)$ is residual signal. Eq. (5) can be represented as:

$$
\begin{gathered}
e(k+1)=\sum_{i=1}^{p} \sum_{j=1}^{p} \mu_{i} \mu_{j}\left[\left(\bar{A}_{i j}-\Delta L_{i} C_{j}\right) e(k)+\left(\bar{E}_{n, i j}-\Delta L_{i} F_{n, j}\right) n(k)\right. \\
\left.+\left(\bar{E}_{f, i j}-\Delta L_{i} F_{f, j}\right) f(k)\right] \\
r(k)=\sum_{i=1}^{p} \mu_{i}\left[C_{i} e(k)+F_{n, i} n(k)+F_{f, i} f(k)\right],
\end{gathered}
$$

where $\bar{A}_{i j}=A_{i}-L_{i}^{*} C_{j}, \bar{E}_{n, i j}=E_{n, i}-L_{i}^{*} F_{n, j}$ and $\bar{E}_{f, i j}=E_{f, i}-$ $L_{i}^{*} F_{f, j}$. Eq. (6) can be more simplified and represented as

$$
\begin{gathered}
e(k+1)=\sum_{i=1}^{p} \sum_{j=1}^{p} \mu_{i} \mu_{j}\left[\left(\tilde{A}_{i j} e(k)+\tilde{E}_{n, i j} n(k)+\tilde{E}_{f, i j} f(k)\right]\right. \\
r(k)=\sum_{i=1}^{p} \mu_{i}\left[C_{i} e(k)+F_{n, i} n(k)+F_{f, i} f(k)\right],
\end{gathered}
$$

where $\tilde{A}_{i j}=\bar{A}_{i j}-\Delta L_{i} C_{j}, \tilde{E}_{n, i j}=\bar{E}_{n, i j}-\Delta L_{i} F_{n, j}$ and $\tilde{E}_{f, i j}=$ $\bar{E}_{f, i j}-\Delta L_{i} F_{f, j}$.

\subsection{Residual Evaluation}

After the design of the residual generator, the remaining important task for robust fault detection is the evaluation of the generated residual. Based on [10], threshold value $J_{t h}>0$ can be calculated. Using the following logic relationship for fault detection:

$\|r(k)\|_{2, N}<J_{t h} \quad$ no fault

$\|r(k)\|_{2, N}<J_{t h} \quad$ fault,

where the so-called residual evaluation $\|r(k)\|_{2, N}$ is determined by

$\|r(k)\|_{2, N}=\left[\sum_{k=0}^{N} r^{T}(k) r(k)\right]^{\frac{1}{2}}$,

with $N$ is length of the evaluated window. Since an evaluation of the signal over the whole time range is impractical, it is desired that the fault will be detected as easy as possible. Based on (7), we have $\|r(k)\|_{2, N}=\| r_{n}(k)+$ $r_{f}(k) \|_{2, N}$ where $r_{n}(k)$ and $r_{f}(k)$ are defined as: $r_{n}(k)=\left.r(k)\right|_{f(k)=0}$, $r_{f}(k)=\left.r(k)\right|_{n=0}$. Moreover, the fault-free case residual evaluation function is $\|r(k)\|_{2, N} \leq\left\|r_{n}(k)\right\|_{2, N} \leq J_{t h, n}$, where $J_{t h, n}$ $=\sup _{n \in L_{2}}\left\|r_{n}(k)\right\|_{2, N}$. We choose the threshold $J_{t h}$ as $J_{t h}=J_{t h, n}$. Where $J_{t h}$ is constant and can be evaluated off-line.

\section{ROBUST FAULT DETECTION DESIGN}

Robust fault detection design is shown in the following sub-sections.

\subsection{Gain Matrix Design Based on DARE}

The gain matrix for each local sub-system is obtained. The computation of covariance of residual signal generated by kalman filter-based residual generator and fault detection filter is shown. Consider system (7) with $\Delta L_{i}=0$, the following system is obtained.

$$
\begin{gathered}
e(k+1)=\sum_{i=1}^{p} \sum_{j=1}^{p} \mu_{i} \mu_{j}\left[\left(\bar{A}_{i j} e(k)+\left(\bar{E}_{n, i j} n(k)+\bar{E}_{f, i j} f(k)\right]\right.\right. \\
r(k)=\sum_{j=1}^{p} \mu_{i}\left[C_{i} e(k)+F_{n, i} n(k)+F_{f, i} f(k)\right] \\
r(k)=r_{n}(k)+r_{f}(k),
\end{gathered}
$$

Based on [11], the following theorem provides a solution to obtain $L_{i}^{*}$, the proof for linear system is given in [12].

Theorem 1. Each sub-system is stable and satisfy $H_{\infty}-$ norm if 
$L_{i}^{*}=\left(S+A_{i} P C_{i}^{T}\right)\left(C_{i} P C_{i}^{T}+R\right)^{-1}$,

where $Q=E_{n, i} \Sigma_{n} E_{n, i}^{T}, R=F_{n, i} \Sigma_{n} F_{n, i}^{T}, S=E_{n, i} \Sigma_{n} F_{n, i}^{T}$ and $P \geq$ 0 is the covariance of the estimation error, it is given as a solution of the following DARE

$$
P=A_{i} P A_{i}^{T}+Q-\left(S+A_{i} P C_{i}^{T}\right)\left(C_{i} P C_{i}^{T}+R\right)^{-1}\left(S+A_{i} P C_{i}^{T}\right)^{T},
$$

\subsection{Covariance of Residual Generated by Kalman Filter}

For FD of the dynamic system with only stochastic noise, the steady-state one-step predictive kalman filter is often used as residual generator $[13,14]$. In this case, the generated residual is a zero-mean white Gaussian signal with minimal covariance in the fault-free case, and the residual covariance can be easily calculated. Based on the statistical characteristic of residual signal, the covariance matrix $\phi_{r}(i)$ of residual $r(k)$ is equal to the covariance matrix of noise induced residual signal $r_{n}(k)$, therefore

$$
\begin{array}{ll}
\phi_{r}(l)=\phi_{r_{n}}(l)=C i P C_{i}^{T}+R & l=0 \\
\phi_{r}(l)=\phi_{r_{n}}(l)=0, & l \neq 0,
\end{array}
$$

Since the residual vector $r_{k-s, k}$ in the evaluated window is defined as $r_{k-s, k}=\left[r^{T}(k-s), \ldots, r^{T}(k)\right]^{T}$ thus the covariance matrix of residual vector $r_{k-s, k}$ is

$$
\begin{gathered}
\sum=E\left\{r_{k-s, k} r_{k-s, k}^{T}\right\} \\
=\left[\begin{array}{cccc}
\phi_{r}(0) & \phi_{r}^{T}(1) & \cdots & \phi_{r}^{T}(S) \\
\phi_{r}(1) & \phi_{r}(0) & \ddots & \vdots \\
\vdots & \ddots & \ddots & 0 \\
\phi_{r}(S) & \cdots & 0 & \phi_{r}(0)
\end{array}\right]_{(s+1) \times(s+1)} \\
=\left[\begin{array}{cccc}
C_{i} P C_{i}^{T}+R & 0 & \cdots & 0 \\
\phi_{r}(1) & \cdots & \ddots & \vdots \\
\vdots & \ddots & \ddots & 0 \\
\phi_{r}(s) & \cdots & 0 & C_{i} P C_{i}^{T}+R
\end{array}\right]_{(s+1) \times(s+1)}
\end{gathered}
$$

Since the generated residual $r(k)$ is un-correlated, it can be found from above expression, the covariance matrix of residual signal $r_{k-s, s}$ is a block diagram matrix, there fore a statistical residual for the residual vector $r_{k-s, k}$ can be easily carried out based on this property.

\subsection{Increment Gain Matrix Design Based on LMI}

Incremented gain matrix $\Delta L_{i}$ shown in (7) is designed. For this purpose, the residual covariance will be firstly analyzed. If the residual dynamic is stable, the unique stabilizing solution of following DARE denoted by $\phi$ is the covariance of estimated error

$$
\begin{gathered}
\lim _{k=\infty} E\left\{e(k+1) e^{T}(k+1)\right\}=\phi \\
=\sum_{i=1}^{p} \mu_{i}^{4}\left\{\left(\bar{A}_{i i}-\Delta L_{i} C_{i}\right) \phi\left(\bar{A}_{i i}-\Delta L_{i} C_{i}\right)^{T}\right. \\
\left.+\left(\bar{E}_{n, i i}-\Delta L_{i} F_{n, i}\right) \sum_{n}\left(\bar{E}_{n, i i}-\Delta L_{i} F_{n, i}\right)\right\} \\
+\frac{1}{4} \sum_{i=1}^{p} \sum_{i<j}^{p} \mu_{i}^{2} \mu_{j}^{2}\left\{\left(\bar{A}_{i j}-\Delta L_{i} C_{j}+\bar{A}_{j i}-\Delta L_{j} C_{i}\right) \phi\right.
\end{gathered}
$$

eq. (15) contd.....

$$
\begin{gathered}
\left(\bar{A}_{i j}-\Delta L_{i} C_{j}+\bar{A}_{j i}-\Delta L_{j} C_{i}\right)^{T} \\
+\left(\bar{E}_{n, i j}-\Delta L_{i} F_{n, j}+\bar{E}_{n, j i}-\Delta L_{j} F_{n, i}\right) \sum_{n} \\
\left.\left(\bar{E}_{n, i j}-\Delta L_{i} F_{n, j}+\bar{E}_{n, j i}-\Delta L_{j} F_{n, i}\right)^{T}\right\}
\end{gathered}
$$

Since the estimated error $e(k+1)$ is independent of $n(k)$, the covariance of residual signal $\phi_{r}$ is

$$
\begin{gathered}
\lim _{k=\infty} E\left\{r(k) r^{T}(k)\right\}=\phi_{r}=\sum_{i=1}^{p} \mu_{i}^{2}\left\{\bar{C}_{i} \phi \bar{C}_{i}^{T}+\bar{F}_{n, i} \sum_{n} \bar{F}_{n, i}^{T}\right\} \\
+\frac{1}{4} \sum_{i=1}^{p} \sum_{i<j} \mu_{i} \mu_{j}\left\{\left(\bar{C}_{i}+\bar{C}_{j}\right) \phi\left(\bar{C}_{i}+\bar{C}_{j}\right)^{T}\right. \\
\left.+\left(\bar{F}_{n, i}+\bar{F}_{n, j}\right) \sum_{n}\left(\bar{F}_{n, i}+\bar{F}_{n, j}\right)^{T}\right\}
\end{gathered}
$$

Therefore,

$$
\begin{gathered}
\operatorname{tr}\left(\phi_{r}\right)=\operatorname{tr}\left(\sum_{i=1}^{p} \mu_{i}^{2} C_{i} \phi C_{i}^{T}+\sum_{i=1}^{p} \mu_{i}^{2} F_{n, i} \sum_{n} F_{n, i}^{T}\right. \\
+\frac{1}{4} \sum_{i=1}^{p} \sum_{i<1}^{p} \mu_{i} \mu_{j}\left(C_{i}+C_{j}\right) \phi\left(C_{i}+C_{j}\right)^{T} \\
\left.\frac{1}{4} \sum_{i=1}^{p} \sum_{i<1}^{p} \mu_{i} \mu_{j}\left(F_{n, i}+F_{n, j}\right) \sum_{n}\left(F_{n, i}+F_{n, j}\right)^{T}\right) \\
=\operatorname{tr}\left(\sum_{i=1}^{p} \mu_{i}^{2} C_{i} \phi C_{i}^{T}\right)+\operatorname{tr}\left(\sum_{i=1}^{p} \mu_{i}^{2} F_{n, i} \sum_{n} F_{n, i}^{T}\right) \\
+\frac{1}{4} \operatorname{tr}\left(\sum_{i=1}^{p} \sum_{i<1}^{p} \mu_{i} \mu_{j}\left(C_{i}+C_{j}\right) \phi\left(C_{i}+C_{j}\right)^{T}\right) \\
+\frac{1}{4} \operatorname{tr}\left(\sum_{i=1}^{p} \sum_{i<1}^{p} \mu_{i} \mu_{j}\left(F_{n, i}+F_{n, j}\right) \sum_{n}\left(F_{n, i}+F_{n, j}\right)^{T}\right)
\end{gathered}
$$

where

$\operatorname{tr}\left(\sum_{i=1}^{p} \mu_{i}^{2} F_{n, i} \sum_{n} F_{n, i}^{T}+\frac{1}{4} \operatorname{tr}\left(\sum_{i=1}^{p} \sum_{i<1}^{p} \mu_{i} \mu_{j}\left(F_{n, i}+F_{n, j}\right) \sum_{n}\left(F_{n, i}+F_{n, j}\right)^{T}\right)\right)$ is only decided by noise and is a positive scalar constant. As $\operatorname{tr}(A B)=\operatorname{tr}(B A)$ then,

$$
\begin{gathered}
\operatorname{tr}\left(\phi_{r}\right)=\operatorname{tr}\left(\sum_{i=1}^{p} \mu_{i}^{2} \phi C_{i} C_{i}^{T}+\sum_{i=1}^{p} \mu_{i}^{2} F_{n, i}^{T} \sum_{n} F_{n, i}^{T}\right. \\
+\frac{1}{4} \sum_{i=1}^{p} \sum_{i<j}^{p} \mu_{i} \mu_{j} \phi\left(C_{i}+C_{j}\right)\left(C_{i}+C_{j}\right)^{T} \\
\left.\frac{1}{4} \sum_{i=1}^{p} \sum_{i<j}^{p} \mu_{i} \mu_{j}\left(F_{n, i}+F_{n, j}\right) \sum_{n}\left(F_{n, i}+F_{n, j}\right)^{T}\right)
\end{gathered}
$$

Based on the above results, the optimization of FD design can be expressed as: Find $\Delta L_{i}$ such that, the residual dynamic (7) is stable and

$$
\begin{gathered}
\operatorname{tr}\left(\sum_{i=1}^{p} \mu_{i}^{2} \phi C_{i}^{T} C_{i}\right) \\
+\frac{1}{4} \operatorname{tr}\left(\sum_{i=1}^{p} \sum_{i<j}^{p} \mu_{i} \mu_{j} \phi\left(C_{i}+C_{j}\right)^{T}\left(C_{i}+C_{j}\right)\right) \rightarrow \min
\end{gathered}
$$


Based on [15], the following lemma is obtained.

Lemma 1. Assume that the matrices Li stabilizes the residual dynamics (7) then

$$
\begin{gathered}
\operatorname{tr}(\psi V)=\operatorname{tr}\left(\sum_{i=1}^{p} \mu_{i}^{2} \varphi C_{i}^{T} C_{i}\right) \\
+\frac{1}{4} \operatorname{tr}\left(\sum_{i=1}^{p} \sum_{i<j}^{p} \mu_{i} \mu_{j} \varphi\left(C_{i}+C_{j}\right)^{T}\left(C_{i}+C_{j}\right)\right)
\end{gathered}
$$

where

$$
\begin{gathered}
V=\sum_{i=1}^{p} \mu_{i}^{4}\left(\bar{E}_{n, i i}-\Delta L_{i} F_{n, i}\right) \sum_{n}\left(\bar{E}_{n, i i}-\Delta L_{i} F_{n, i}\right)^{T} \\
+\frac{1}{4} \sum_{i=1}^{p} \sum_{i<j}^{p} \mu_{i}^{2} \mu_{j}^{2}\left(\bar{E}_{n, i j}-\Delta L_{i} F_{n, j}+\bar{E}_{n, j i}-\Delta L_{j} F_{n, i}\right) \\
\sum_{n}\left(\bar{E}_{n, i j}-\Delta L_{i} F_{n, i}+\bar{E}_{n, j i}-\Delta L_{j} F_{n, i}\right)^{T},
\end{gathered}
$$

and $\psi>0$ is the unique stable solution of DARE

$$
\begin{aligned}
& \psi\left.=\sum_{i=1}^{p} \mu_{i}^{4}\left[\left(\bar{A}_{i i}-\Delta L_{i} C_{i}\right)^{T} \psi\left(\bar{A}_{i i}-\Delta L_{i} C_{i}\right)+C_{i}^{T} C_{i}\right)\right] \\
&+\frac{1}{4} \sum_{i=1}^{p} \sum_{i<j}^{p} \mu_{i}^{2} \mu_{j}^{2}\left[\bar{A}_{i j}-\Delta L_{i} C_{j}+\bar{A}_{j i}-\Delta L_{j} C_{i}\right]^{T} \\
& \psi\left[\bar{A}_{i j}-\Delta L_{i} C_{j}+\bar{A}_{j i}-\Delta L_{j} C_{i}\right]+\left(C_{i}+C_{j}\right)^{T}\left(C_{i}+C_{j}\right) .
\end{aligned}
$$

Proof: From the solution of DARE (15) and (16), we know that

$$
\begin{aligned}
& \operatorname{tr}\left(\sum_{i=1}^{p} \mu_{i}^{2} \phi C_{i}^{T} C_{i}\right)+\frac{1}{4} \operatorname{tr}\left(\sum_{i<j}^{p} \mu_{i} \mu_{j} \phi\left(C_{i}+C_{j}\right)^{T}\left(C_{i}+C_{j}\right)\right) \\
& \left.=\operatorname{tr} \sum_{l=0}^{\infty} \sum_{i=1}^{p} \mu^{4}\left(\bar{A}_{i i}-\Delta L_{i} C_{i}\right)^{l} V\left(\bar{A}_{i i}-\Delta L_{i} C_{j}\right)^{l T} C_{i}^{T} C_{i}\right) \\
& \quad+\frac{1}{4} \operatorname{tr} \sum_{i=1}^{p} \sum_{i<j}^{p} \mu_{i}^{2} \mu_{j}^{2}\left(\bar{A}_{i j}-\Delta L_{i} C_{j}+\bar{A}_{j i}-\Delta L_{j} C_{i}\right)^{l} \\
& \left.V\left(\bar{A}_{i j}-\Delta L_{i} C_{j}+\bar{A}_{j i}-\Delta L_{j} C_{i}\right)^{l T}\left(C_{i}+C_{j}\right)^{T}\left(C_{i}+C_{j}\right)\right) \\
& =\operatorname{tr}\left(\sum_{l=0}^{\infty} \sum_{i=1}^{p} \mu^{4}\left(\bar{A}_{i i}-\Delta L_{i} C_{i}\right)^{l T} C_{i}^{T} C_{i}\left(\bar{A}_{i i}^{l}-\Delta L_{i} C_{j}\right)\right) \\
& +\frac{1}{4} \operatorname{tr} \sum_{i=1}^{p} \sum_{i<j}^{p} \mu_{i}^{2} \mu_{j}^{2}\left(\bar{A}_{i j}-\Delta L_{i} C_{j}+\bar{A}_{j i}-\Delta L_{j} C_{i}\right)^{l T} \\
& \left(C_{i}+C_{j}\right)^{T}\left(C_{i}+C_{j}\right)\left(\bar{A}_{i j}-\Delta L_{i} C_{j}+\bar{A}_{j i}-\Delta L_{j} C_{i}\right)^{l} V=\operatorname{tr} \psi V
\end{aligned}
$$

Based on lemma 1, the following theorem is used to obtain the change in gain matrix $\Delta L_{i}$.

Theorem 2. Assume that $\Delta L_{i}$ is given and there exists a symmetric matrix $X>0$ and $\gamma>0$ such that:

1. $\gamma^{2} I+\Delta L_{i}^{T} X \Delta L_{i}>0$

2. $4 \gamma^{2} I-\left(\Delta L_{i}+\Delta L_{j}\right)^{T} X\left(\Delta L_{i}+\Delta L_{j}\right)>0$

3. $-X+\tilde{A}_{i i}^{T} X \tilde{A}_{i i}+C_{i}^{T} C_{i}+\tilde{A}_{i i}^{T} X \Delta L_{i}\left(\gamma^{2} I-\Delta L_{i}^{T} X \Delta L_{i}\right)^{-1}$ $\Delta L_{i}^{T} X \tilde{A}_{i i}<0$
4. $-4 X+\left(\left(\tilde{A}_{i j}+\tilde{A}_{j i}\right)^{T} X\left(\tilde{A}_{i j}+\tilde{A}_{j i}\right)+\left(C_{i}+C_{j}\right)^{T}\left(C_{i}+C_{j}\right)\right.$ $+\left(\tilde{A}_{i j}+\tilde{A}_{j i}\right)^{T} X\left(\Delta L_{i}+\Delta L_{j}\right)\left(\gamma^{2} I-\left(\Delta L_{i}+\Delta L_{j}\right)^{T} X\left(\Delta L_{i}+\right.\right.$ $\left.\left.\Delta L_{j}\right)\right)^{-1}\left(\Delta L_{i}+\Delta L_{j}\right)^{T} X\left(\tilde{A}_{i j}+\tilde{A}_{j i}\right)<0$,

are satisfied for $\forall \mathrm{I}, \mathrm{j}$. Then:

i)

system (7) is stable

ii)

$$
\Psi \leq X \text { consequently } \operatorname{tr}\left(\sum_{i=1}^{p} \mu_{1}^{2} \phi C_{i}^{T} C_{i}\right)+
$$

$$
\frac{1}{4} \operatorname{tr}\left(\sum_{i=1}^{p} \sum_{i<j}^{p} \mu_{i} \mu_{j} \phi\left(C_{i}+C_{j}\right)^{T}\left(C_{i}+C_{j}\right)\right) \leq \operatorname{tr}(X V) .
$$

Proof: The results (i) follows directly from the bounded real lemma [16]. For the results (ii), based on the schur complements lemma, condition (3) is equivalent to the following LMI:

$$
\left[\begin{array}{cc}
-\gamma^{2} I+\Delta L_{i}^{T} X \Delta L_{i} & \Delta L_{i}^{T} X \tilde{A}_{i i} \\
\tilde{A}_{i i}^{T} X \Delta L_{i} & -X+\tilde{A}_{i i}^{T} X \tilde{A}_{i i}+C_{i}^{T} C_{i}
\end{array}\right]<0,
$$

for $1 \leq i \leq p$ hold if $X>\tilde{A}_{i i}^{T} X \tilde{A}_{i i}+C_{i}^{T} C_{i}$. And condition (4) is equivalent to the following LMI:

$$
\begin{aligned}
& {\left[-4 \gamma^{2} l+\left(\Delta L_{i}+\Delta L_{j}\right)^{T} X\left(\Delta L_{i}+\Delta L_{j}\right)\right.} \\
& \left(\tilde{A}_{i j}+\tilde{A}_{j i}\right)^{T} X\left(\Delta L_{i}+\Delta L_{j}\right) \\
& \left(\Delta L_{i}+\Delta L_{j}\right)^{T} X\left(\tilde{A}_{i j}+\tilde{A}_{j i}\right) \\
& \left.\left[\begin{array}{c}
-4 X+\left(\tilde{A}_{i j}+\tilde{A}_{j i}\right) X\left(\tilde{A}_{i j}+\tilde{A}_{j i}\right) \\
+\left(C_{i}+C_{j}\right)^{T}\left(C_{i}+C_{j}\right)
\end{array}\right]\right]
\end{aligned}
$$

for $1 \leq i \leq p$ hold if

$$
4 X>\left(\tilde{A}_{i j}+\tilde{A}_{j i}\right)^{T} X\left(\tilde{A}_{i j}+\tilde{A}_{j i}\right)+\left(C_{i}+C_{j}\right)^{T}\left(C_{i}+C_{j}\right) .
$$

Compering (22) and (23) with DARE (21), based on the monotonicity of the DARE [17], we know $\Psi \leq X$. And based on Lemma 1, we have that

$$
\begin{gathered}
\operatorname{tr}\left(\sum_{i=1}^{p} \mu_{i}^{2} \phi C_{i}^{T}\right)+\frac{1}{4} \operatorname{tr}\left(\sum_{i=1}^{p} \sum_{i<j}^{p} \mu_{i} \mu_{j} \phi\left(C_{i}+C_{j}\right)^{T}\right. \\
\left.\left(C_{i}+C_{j}\right)\right)=\operatorname{tr}(\Psi V) \leq \operatorname{tr}(X V)
\end{gathered}
$$

Based on Theorem 2, the previous problem can be reformulated as: for a given $\gamma>0$ and symmetric $X>0$, find $\Delta L_{i}$ such that:

$$
\begin{aligned}
& -X+\tilde{A}_{i i}^{T} X \tilde{A}_{i i}+C_{i}^{T} C_{i} \\
& +\tilde{A}_{i i}^{T} X \Delta L_{i}\left(\gamma^{2} I-\Delta L_{i}^{T} X \Delta L_{i}\right)^{-1} \Delta L_{i}^{T} X \tilde{A}_{i i}<0, \\
& \text { and } \operatorname{tr}(X V) \rightarrow \min \text { for } 1 \leq i \leq p \\
& -4 X+\left(\tilde{A}_{i j}+\tilde{A}_{j i}\right)^{T} X\left(\tilde{A}_{i j}+\tilde{A}_{j i}\right)+\left(C_{i}+C_{j}\right)^{T}\left(C_{i}+C_{j}\right) \\
& \qquad+\left(\tilde{A}_{i j}+\tilde{A}_{j i}\right)^{T} X\left(\Delta L_{i}+\Delta L_{j}\right)\left(\gamma^{2} I-\left(\Delta L_{i}+\Delta L_{j}\right)^{T}\right. \\
& \left.\qquad X\left(\Delta L_{i}+\Delta L_{j}\right)\right)^{-1}\left(\Delta L_{i}+\Delta L_{j}\right)^{T} X\left(\tilde{A}_{i j}+\tilde{A}_{j i}\right)<0,
\end{aligned}
$$


and $\operatorname{tr}(X V) \rightarrow$ min. for $1 \leq i<j \leq p$. It is known that

$$
\operatorname{tr}(X V)=\operatorname{tr} \sum_{n}^{1 / 2}\left(\bar{E}_{n, i i}-\Delta L_{i} F_{n, i}\right)^{T} X\left(\bar{E}_{n, i i}-\Delta L_{i} F_{n, i}\right) \sum_{n}^{1 / 2},
$$

for $1 \leq i \leq p$ and

$$
\begin{gathered}
4 \operatorname{tr}(X V)=\operatorname{tr} \sum_{n}^{1 / 2}\left(\left[\bar{E}_{n, i j}-\Delta L_{i} F_{n, j}\right]+\left[\bar{E}_{n, j i}-\Delta L_{j} F_{n, i}\right]\right)^{T} \\
X\left(\left[\bar{E}_{n, i j}-\Delta L_{i} F_{n, j}\right]+\left[\bar{E}_{n, j i}-\Delta L_{j} F_{n, i}\right]\right) \sum_{n}^{1 / 2},
\end{gathered}
$$

for $1 \leq i<j \leq p$. Therefore the minimization of $\operatorname{tr}(X V)$, can be realized with the following method,

$$
\sum_{n}^{1 / 2}\left(\bar{E}_{n, i i}^{T}-\Delta L_{i} F_{n, i}\right) X\left[\bar{E}_{n, i i}-\Delta L_{i} F_{n, i}\right) \sum_{n}^{1 / 2}<\bar{\phi},
$$

for $1 \leq i \leq p$, and

$$
\begin{aligned}
& \sum_{n}^{1 / 2}\left(\left[\bar{E}_{n, i j}-\Delta L_{i} F_{n, j}\right]+\left[\bar{E}_{n, j i}-\Delta L_{j} F_{n, i}\right]\right)^{T} \\
& X\left(\left[\bar{E}_{n, i j}-\Delta L_{i} F_{n, j}\right]+\left[\bar{E}_{n, j i}-\Delta L_{j} F_{n, i}\right) \sum_{n}^{1 / 2}\right.
\end{aligned}
$$

$1 \leq i \leq j \leq p$.

This formulation can be represented as LMI as:

$$
\left[\begin{array}{cc}
\bar{\phi} & S\left(\bar{E}_{n, i i}^{T} X-F_{n, i}^{T} Y_{i}^{T}\right) \\
* & X
\end{array}\right]>0
$$

for $1 \leq i \leq p$, and

$$
\left[\begin{array}{cc}
4 \bar{\phi} & S\left(\bar{E}_{n, i j}^{T} X-F_{n, j}^{T} Y_{i}^{T}+\bar{E}_{n, j i}^{T} X-F_{n, i}^{T} Y_{j}^{T}\right) \\
* & X
\end{array}\right]>0
$$

for $1 \leq i<j \leq p$,

where $S=\sum_{n}^{1 / 2}, Y_{i}=X \Delta L_{i}$. Therefore, this problem can be reformulated as the following optimization problem:

For a given $\gamma>0$, find symmetric matrices $X>0$ and matrix $Y$, so that the following LMIs:

$$
\begin{aligned}
& {\left[\begin{array}{cccc}
-X & X \bar{A}_{i i}-Y_{i} C_{i} & Y_{i} & 0 \\
* & -X & 0 & C_{i}^{T} \\
* & * & -l & 0 \\
* & * & * & -\gamma^{2} l
\end{array}\right]<0,} \\
& {\left[\begin{array}{cc}
\bar{\phi} & S\left(\bar{E}_{n, i i}^{T} X-F_{n, i}^{T} Y_{i}^{T}\right) \\
* & X
\end{array}\right]>0}
\end{aligned}
$$

for $1 \leq i \leq p$, and

$$
\begin{aligned}
& {\left[\begin{array}{cccc}
-4 X & M(i j) & Y_{i}+Y_{j} & 0 \\
* & -X & 0 & \left(C_{i}+C_{j}\right)^{T} \\
* & * & -l & 0 \\
* & * & * & -4 \gamma^{2} l
\end{array}\right]<0,} \\
& {\left[\begin{array}{cc}
4 \bar{\phi} & S\left(\bar{E}_{n, i j}^{T} X-F_{n, j}^{T} Y_{i}^{T}+\bar{E}_{n, j i}^{T} X-F_{n, i}^{T} Y_{j}^{T}\right) \\
* &
\end{array}\right]>0,}
\end{aligned}
$$

for $1 \leq i<j \leq p$ where

$\mathrm{M}(\mathrm{ij})=X \bar{A}_{i j}-Y_{i} C_{j}+X \bar{A}_{j i}-Y_{j} C_{i}$. Then the problem can be solved and the solution for $\Delta L_{i}$ is $\Delta L_{i}=X^{-1} Y_{i}$.

\section{LATERAL VEHICLE DYNAMIC MODEL}

In recent years many research have been done in the field of vehicle dynamics, many achievements have been fulfilled [18-20]. And in many applications different vehicle dynamic models have been achieved. The derivation of the vehicle dynamic model is based on the physical motion equations, therefore the different models can be classified according to the quality of model's freedom. The general used one-track model (or bicycle model) is a 3 DOF model [21], for the vehicle is simplified as a whole mass with the center of gravity on the ground, which can only move in $x$ axis, $y$ axis, and yaw around $z$ axis. The coordinate system is shown in

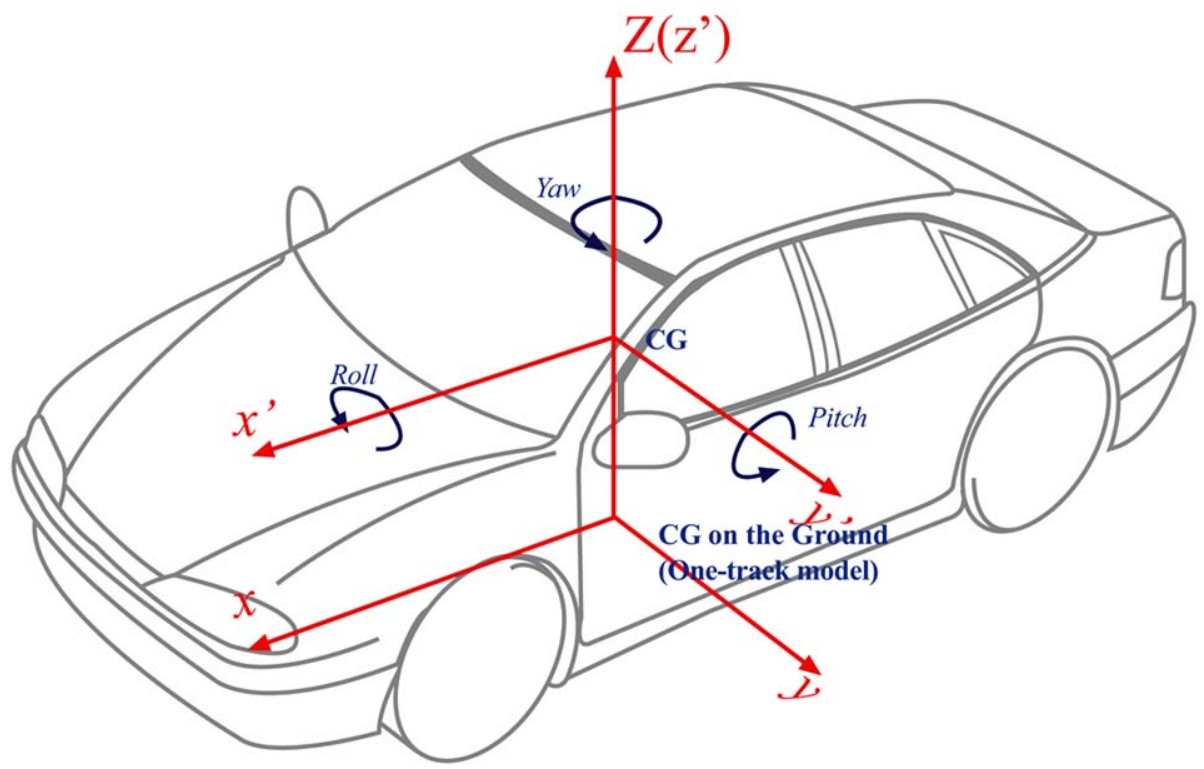

Fig. (1). Coordinate system of vehicle model. 
Fig. (1), which is fixed to the CG. For the purpose of studying the roll motion of the vehicle, the $\mathrm{CG}$ is not assumed on the ground. Comparing with one track model, the roll motion around the $x$-axis is introduced, so it is called a 4 DOF model. For a more precise description of the vehicle dynamic, the vehicle is modeled as a multi-body system. Some large DOF models have been constructed, such as the vehicle simulation software Trucksim which includes a 14 DOF model. But such kind of model is too complicated to be used for the on-line application, only suitable for some offline or simulation application.

In IFATIS project [21], in order to establish a design framework of model based monitorinng system for vehicle lateral dynamics control systems, the 4 DOF model and onetrack model have been studied. In this paper, one-track model is used. Because of TS fuzzy model can be used with time varing systems so TS fuzzy model can be obtained for Vehicle Lateral Dynamic model.

\subsection{Simulation Results}

Sensor fault for Lateral vehicle dynamic model is studied. Lateral acceleration sensor fault and Yaw rate sensor fault with stochastic noises are detected. In this case, discrete TS fuzzy model is used. After the discretization of each sub system, using 10 milliseconds as sampling time, the vehicle lateral dynamic model is represented ba the following:

$$
\begin{gathered}
x(k+1)=\sum_{i=1}^{4} \mu_{i}\left[A_{i} x(k)+B_{i}\left(\delta_{L}^{*}(k)+n_{\delta_{L}}(k)\right)+E_{f, i} f(t)\right] \\
x(k)=\sum_{i=1}^{4} \mu_{i}\left[C_{i} x(k)+D_{i} \delta_{L}^{*}(k)+v(k)+F_{f, i} f(k)\right] \\
V(k)=\left[\begin{array}{ll}
1 & 0 \\
0 & 1
\end{array}\right]\left[\begin{array}{c}
n_{a y}(k) \\
n_{r}(k)
\end{array}\right] \\
A_{1}=\left[\begin{array}{ll}
0.7512 & 0.0099 \\
0.2181 & 0.7118
\end{array}\right], \quad B_{1}=E_{f, 1}\left[\begin{array}{l}
0.0941 \\
0.3598
\end{array}\right]
\end{gathered}
$$

where

$$
\begin{aligned}
& A_{2}=\left[\begin{array}{cc}
0.7486 & -0.0072 \\
0.2178 & 0.7093
\end{array}\right], \quad B_{2}=E_{f, 2}\left[\begin{array}{l}
0.0901 \\
0.3594
\end{array}\right] \\
& A_{3}=\left[\begin{array}{cc}
0.9761 & 0.0132 \\
0.2904 & 0.9714
\end{array}\right], \quad B_{3}=E_{f, 3}\left[\begin{array}{l}
0.0122 \\
0.4048
\end{array}\right] \\
& A_{4}=\left[\begin{array}{cc}
0.9727 & -0.0095 \\
0.29 & 0.968
\end{array}\right], \quad B_{4}=E_{f, 4}\left[\begin{array}{l}
0.0075 \\
0.4043
\end{array}\right]
\end{aligned}
$$

\subsection{Residual Generator Design}

As introduced above, the residual for nonlinear system is represented by TS fuzzy filter of the form like

$$
\begin{gathered}
\hat{x}(k+1)=\sum_{i=1}^{4} \mu_{i}\left[A_{i} \hat{x}(k)+B_{i} u(k)+\left(L_{i}^{*}+\Delta L_{i}\right)(y(k)-\hat{y}(k))\right] \\
\hat{y}(k)=\sum_{i=1}^{4} \mu_{i}\left[C_{i} \hat{x}(k)+D_{i} u(k)\right]
\end{gathered}
$$

where $L_{i}$ and $\Delta L_{i}$ are defined as in (7). The following are the details of the sub models and corresponding filter-based residual generators.

\section{The First Sub Model}

In this case, the steering angle is taken as input signal, and lateral acceleration as output signal. The residual generated is

$r_{1}=a_{y}-\hat{a}_{y}$ are

The gain matrices obtained from solving the DARE (11)

$$
\begin{aligned}
& L_{1}^{*}=\left[\begin{array}{l}
0.0012 \\
0.0067
\end{array}\right], \quad L_{2}^{*}=\left[\begin{array}{l}
0.0012 \\
0.0067
\end{array}\right], \\
& L_{3}^{*}=\left[\begin{array}{l}
-0.0003 \\
-0.0027
\end{array}\right], \quad L_{4}^{*}=\left[\begin{array}{l}
0.0001 \\
0.0072
\end{array}\right]
\end{aligned}
$$

The increment in gain matrices are obtained by solving (28) $-(31)$

$$
\begin{aligned}
& \Delta L_{1}=\left[\begin{array}{c}
-0.0053 \\
0.0056
\end{array}\right], \quad \Delta L_{2}=\left[\begin{array}{c}
-0.0055 \\
0.0056
\end{array}\right], \\
& \Delta L_{3}=\left[\begin{array}{c}
-0.0054 \\
-0.0192
\end{array}\right], \quad \Delta L_{4}=\left[\begin{array}{c}
-0.0067 \\
-0.072
\end{array}\right]
\end{aligned}
$$

The covariance matrices for each sub-system based on (14) are

$$
\begin{aligned}
\sum_{n, 1} & =\left[\begin{array}{ccc}
390.1355 & 0 & \cdots \\
0 & 390.1355 & \ddots \\
0 & 0 & \cdots
\end{array}\right]_{21 \times 21} \\
\sum_{n, 2} & =\left[\begin{array}{ccc}
343.8649 & 0 & \cdots \\
0 & 343.8649 & \ddots \\
0 & 0 & \cdots
\end{array}\right]_{21 \times 21} \\
\sum_{n, 3} & =\left[\begin{array}{ccc}
8.0357 & 0 & \cdots \\
0 & 8.0357 & \ddots \\
0 & 0 & \cdots
\end{array}\right]_{21 \times 21} \\
\sum_{n, 4} & =\left[\begin{array}{ccc}
6.4026 & 0 & \cdots \\
0 & 6.4026 & \ddots \\
0 & 0 & \cdots
\end{array}\right]_{21 \times 21}
\end{aligned}
$$

\section{The Second Sub Model}

In this case, the steering angle is adopted as input signal, yaw rate as output signal, the residual generated is

$r_{2}=r-\hat{r}$ 
(a)

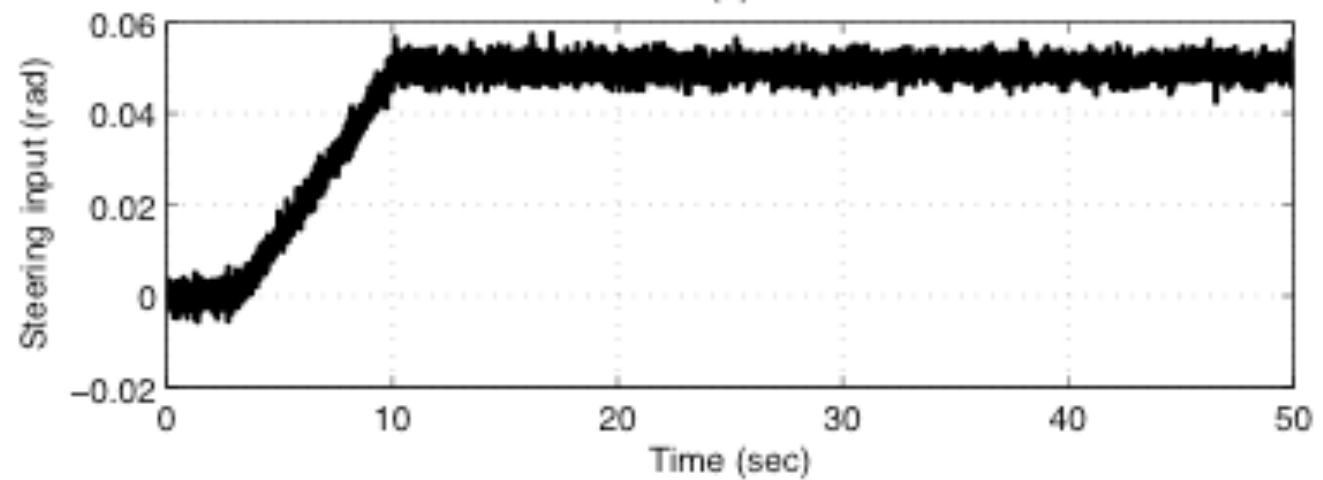

(b)

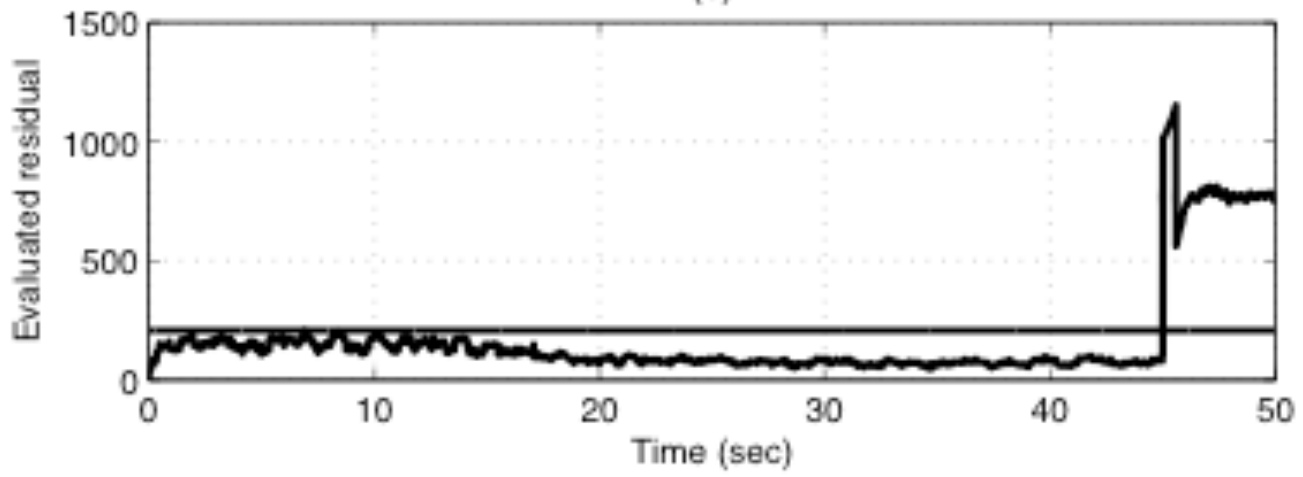

Fig. (2). Robust fault detection for lateral acceleration with stochastic noises. are

The gain matrices obtained from solving the DARE (11)

$$
\begin{array}{ll}
L_{1}^{*}=\left[\begin{array}{l}
0.1298 \\
0.4805
\end{array}\right], \quad L_{2}^{*}=\left[\begin{array}{l}
0.1107 \\
0.4756
\end{array}\right], \\
L_{3}^{*}=\left[\begin{array}{c}
-0.0998 \\
0.6853
\end{array}\right], \quad L_{4}^{*}=\left[\begin{array}{c}
0.0034 \\
0.677
\end{array}\right]
\end{array}
$$

The increment in gain matrices are obtained by solving (28) $-(31)$

$$
\begin{array}{cc}
\Delta L_{1}=\left[\begin{array}{c}
-0.0998 \\
0.2443
\end{array}\right], \quad \Delta L_{2}=\left[\begin{array}{c}
-0.0975 \\
0.2509
\end{array}\right], \\
\Delta L_{3}=\left[\begin{array}{l}
0.0079 \\
0.2417
\end{array}\right], \quad \Delta L_{4}=\left[\begin{array}{l}
0.0151 \\
0.2598
\end{array}\right]
\end{array}
$$

The covariance matrices for each sub-system based on (14) are

$$
\begin{aligned}
& \sum_{1}=\left[\begin{array}{ccc}
0.5798 & 0 & \cdots \\
0 & 0.5798 & \ddots \\
0 & 0 & \cdots
\end{array}\right]_{21 \times 21} \\
& \sum_{2}=\left[\begin{array}{ccc}
0.5784 & 0 & \cdots \\
0 & 0.5784 & \ddots \\
0 & 0 & \cdots
\end{array}\right]_{21 \times 21}
\end{aligned}
$$

$$
\begin{aligned}
& \sum_{3}=\left[\begin{array}{ccc}
0.7317 & 0 & \cdots \\
0 & 0.7317 & \ddots \\
0 & 0 & \cdots
\end{array}\right]_{21 \times 21} \\
& \sum_{4}=\left[\begin{array}{ccc}
0.7297 & 0 & \cdots \\
0 & 0.7297 & \ddots \\
0 & 0 & \cdots
\end{array}\right]_{21 \times 21}
\end{aligned}
$$

\subsection{Residual Evaluation}

After the design of the residual generator, the remaining important task for robust fault detection is the residual evaluator. The residual evaluation consists of evaluation function and threshold value. Using $L_{2}-$ norm as evaluation function with the length of evaluation window $N=20$. The threshold value is calculated at fault free case.

\section{The First Sub Model}

The known input (steering angle) with noise is shown in Fig. (2a). The data with an offset sensor fault of $5 \mathrm{~m} / \mathrm{s}^{2}$ occured at $t=48$ second is used to validate the designed robust FD system. The threshold value in this case is $J_{t h}=$ 207.1923. In Fig. (2b), from $t=48$ second the evaluated signal has exceeded the threshold value.

\section{The Second Sub Model}

The known input (steering angle) with noise is shown in Fig. (3a). The data with an offset sensor fault of $5 \mathrm{~m} / \mathrm{s}^{2}$ occured at $t=44$ second are used to validate the designed 
(a)

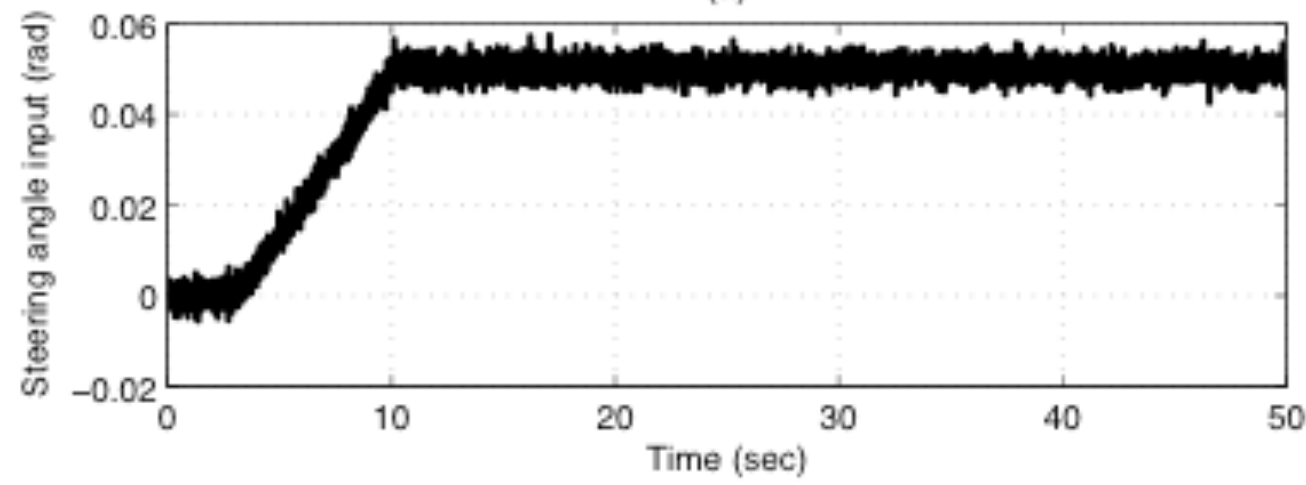

(b)

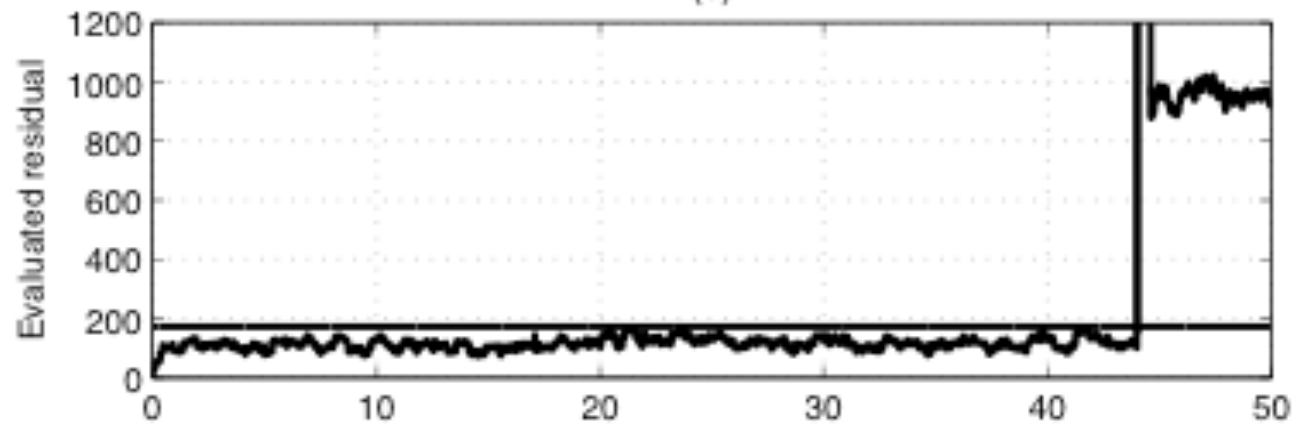

Fig. (3). Robust fault detection for yaw rate with stochastic noise.

robust FD system. The threshold value in this case is $J_{t h}=$ 172.3031. In Fig. (3b), from $t=44 \mathrm{sec}$ and the evaluated signal has exceeded the threshold value.

\section{CONCLUSIONS}

In this paper, robust FD approach for non-linear system with measurement noises has been developed. The nonlinear system is represented by TS fuzzy model. The generated algorithm consists of two parts, in the first part, the fault detection for each fuzzy subsystem is obtained by solving DARE, in the second part, the incremented fault detection is obtained from reducing covariance matrix of residual signal. The generated FD system is robust against stochastic noises and sensitive to the fault. The design procedure has been provided in term of LMIs.

\section{REFERENCES}

[1] S.X. Ding, P. Zhang, P.M. Frank and M. Sader, "Multiobjective design of fault detection filters", ECC'03, Technical Session 4, Cambridge, UK, 2003.

[2] D. Simon, "Kalman filter for fuzzy discrete time dynamic systems", Applied Soft Comuting, vol. 3, pp. 191-207, 2003.

[3] T. Takagi and M. Sugeno, "Fuzzy identification of systems and its applications to modelling and control", IEEE Tranaction on System Man and Cybernetics, vol. 15, no. 1, pp 166-132, 1985.

[4] M.G. El-ghatwary, S.X. Ding and Z. Gao, "Robust fault detection for uncertain Takagi-Sugeno fuzzy systems with parametric uncertainty and process disturbances", Proceeding of IFAC Symposium SAFE PROCESS, Beijing, China, 2006.

[5] Z. Gao, X. Shi and S.X. Ding, "Observer design for T-S fuzzy systems with measurment output noises", IFAC World Control Congress, Prague, 2005.
[6] Z. Gao, T. Chai and H. Wang, "A robust fault detection filter for stochastic system via descriptor estimator and parametric gain design", IET Proceedings Control Theory Applications, vol. 1, no. 5, pp. 1286-1293, 2007.

[7] Z. Gao and S.X. Ding, "Sensor fault reconstruction and compensation for a class of nonlinear state-space systems via descriptor system approach", IET Proceedings Control Theory Applications, vol. 1, no. 3, pp. 578-585, 2007.

[8] N. Zhang, C. Donald and I. Wunsch, "An extended Kalman filter (EKF) approach on fuzzy system optimization problem", IEEE International Conferance of Fuzzy Systems, 2003, pp. 1465-1470.

[9] Z. Gao, S.X. Ding and Y. Ma, "Robust fault estimation approach and its application in vehicle lateral dynamic systems", Optimal control Applications and Methods, vol. 28, no. 3, pp. 143-156, 2007.

[10] S.X. Ding, P. Zhang and P.M. Frank, "Threshold calculation using lmi-technique and its integration in the design of fault detection systems", Proceedings of the $42^{\text {nd }}$ IEEE Conference On Decision and Control, 2003, pp. 469-474.

[11] Y. Ma, "Integrated design of observer-based fault diagnosis systems and its application to vehicle lateral dynamic control systems"AKS, Duisburg, German, 2006.

[12] M. Zhong, S.X. Ding, B. Tang and J. Lam, "An optimization approach to fdf design for uncertain discrete-time systems", Proceedings $15^{\text {th }}$ IFAC World Congress, 2002.

[13] M. Basseville and I.V. Nikiforov, "Detection of abrupt changes. theorem and application", Prentice Hall, Inc., 1993.

[14] S.X. Ding, P. Zhang, B. Huang, E.L. Ding and P.M. Frank, "An approach to norm and statistical methods based residual evaluation", $10^{\text {th }}$ International Conference on Methods and Models in Automation and Robotics, 2004.

[15] Y. Ma and S.X. Ding, "Integrated design of fault detection system with multi-objective optimization", Proceedings of IFAC Symposium, SAFEPROCESS, Beijing, China, 2006.

[16] K. Zhou, "Essential of robust control", Prentice-Hall, Inc, 1998.

[17] S.X. Ding, P.M. Frank, E.L. Ding and T. Jeinsch, "A unified approach to the optimization of fault detection systems", International Journal of Adaptive Control and Signal Processing, vol. 14 , pp. 725-745, 1988. 
[18] T.D. Gillespie, "Fundamentals of vehicle dynamics", Society of Automatic Engineers, Warrendale, USA, 1992.

[19] M. Borner and R. Iserman, "Adaprive one-track model for critical lateral driving situations", In International Synposium on Adavanced Vehicle Control (AVEC), Japan, 2002.
[20] Y. Fukada, "Slip-Angle estimation for vehicle stability control", Vehicle System Dynamics, vol. 32, no, 4, pp. 375-388, 1999.

[21] D. Arnt, "IFATIS Vehicle Models of WP8", Technical Reprot, IFATIS, 2002.

Received: December 05, 2008

(C) El-ghatwary and Ding; Licensee Bentham Open.

This is an open access article licensed under the terms of the Creative Commons Attribution Non-Commercial License (http://creativecommons.org/licenses/by$\mathrm{nc} / 3.0 /$ ), which permits unrestricted, non-commercial use, distribution and reproduction in any medium, provided the work is properly cited. 